\title{
Adaptation of the Masseter and Temporalis Muscles Following Alteration in Length, With or Without Surgical Detachment
}

\author{
LEO C. MAXWELL, DAVID S. CARLSON, JAMES A. MCNAMARA, JR., AND JOHN \\ A. FAULKNER \\ Departments of Physiology (L.C.M., J.A.F.) and Anatomy (D.S.C., J.A.M.), and The \\ Center for Human Growth and Development (L.C.M., D.S.C., J.A.M.), University of \\ Michigan, Ann Arbor, Michigan 48109
}

\begin{abstract}
Histochemical properties, muscle fiber cross-sectional area, muscle fiber length, and the oxidative capacity of masticatory muscles of female rhesus monkeys were assessed following alteration in functional length by an intraoral appliance or by detachment of the muscle. Experimental groups received the appliance only (A); the appliance and subsequent detachment of the masseter (AD); the appliance and detached masseter, but with surgical reattachment of the masseter to the pterygomasseteric sling (ADR); no appliance, but detachment and reattachment of masseter (DR); or an appliance which was removed after 24 weeks to study posttreatment responses (PT). Animals were sacrificed and the muscles were studied at intervals from 4 to 48 weeks after initiation of the experimental period. The results of these studies led to the following conclusions: (1) Stretching the masseter and temporalis muscles within physiological limits did not significantly alter the proportion of fiber types, although oxidative capacity of the fibers was reduced. (2) Fibers with "intermediate" myofibrillar ATPase activity were no more prevalent in experimental than control muscles. (3) The cross-sectional area of Type I fibers of masseter muscles decreased following some experimental procedures, indicating that recruitment of these fibers is the most sensitive to altered jaw function. (4) Minimal alteration of muscle capillarity was induced by any of the experimental procedures. (5) The lengths of masseter muscle fibers in Group PT and of temporalis muscle fibers in groups AD and ADR were greater than in control animals.
\end{abstract}

Skeletal muscle fibers may undergo both morphological and physiological adaptation during growth and in response to altered function due to changes in length, position, and behavior. Morphologically, alteration in functional demand could lead to changes in muscle fiber geometry, cross-sectional area, the amount and distribution of connective tissue within the muscle and its terminal ends, and the length of the individual muscle fibers due to either an addition or deletion of sarcomeres. Physiologically, fibers of limb skeletal muscles have been shown to undergo adaptive change with respect to oxidative capacity, and there is evidence to suggest that fibers may undergo conversion between Type I and Type II myosins during growth or following alteration of function (Maxwell et al., 1979, 1980a).
Nearly all previous studies of muscle adaptation following alteration of function have been undertaken in mammalian limb muscles. There is relatively little known concerning the effects of alteration of function on the muscles of mastication. This is especially unfortunate since clinicians routinely alter the length, position, and habitual function of the perioral muscles and the muscles of mastication during the course of orthodontic treatment and surgical intervention in both growing and adult individuals. The capability of the muscles of

Dr. Maxwell's current address is University of Texas Health Science Center at San Antonio, Department of Physiology, 7703 Floyd Curl Drive, San Antonio, Texas 78284.

Received December 10, 1979; accepted November 7, 1980. 
mastication and perioral muscles to adapt morphologically and physiologically to alterations in function concomitant with muscle lengthening and/or detachment following repositioning of skeletal elements is not known at this time, nor is it clear what effect the adaptation of these muscles has upon posttreatment skeletal relapse which often occurs following therapeutic intervention for the correction of dental skeletal disharmony (Christiansen, 1975; McNamara et al., 1978).

The purpose of this study is to determine the nature and degree of adaptation which takes place within the superficial masseter and temporalis muscles in response to alterations in the functional length or surgical detachment of these muscles. Specifically, we determined alterations relative to control muscles in the histochemical properties, fiber cross-sectional area, fiber length, and oxidative capacity of the masseter muscle and the temporalis muscle in response to altered functional length, with or without myotomy of the masseter muscle.

\section{EXPERIMENTAL DESIGN}

\section{Appliance design}

An intraoral appliance was developed to produce an alteration in length of the muscles of mastication without the necessity of surgical intervention (McNamara, 1975). An occlusal splint which displaced the mandible inferoposteriorly was cast in Ticonium and cemented to the maxillary arch. The effect of the splint was to open the bite approximately $2 \mathrm{~cm}$ incisally, thereby bilaterally stretching the masseter, medial pterygoid, and temporalis muscles. The appliance caused a $15 \%$ lengthening of masseter (Carlson, 1977) and temporalis (unpublished data). The animals adapted readily to the bite-opening appliance and experienced no significant problems in feeding behavior.

\section{Experimental groups}

Thirty-one adult female rhesus monkeys (Macaca mulatta) were assigned to five experimental groups. Each of the monkeys exhibited a full permanent dentition, with the exception of incomplete eruption of the third molars in some cases, indicating that each was at least 4.5 years of age (Hurme and Van Wagenen, 1961). Previously published data from the muscles of seven adult female rhesus monkeys (Maxwell et al., 1979) were used for compar- ison with experimental results. Experimental groups were defined in order to evaluate (1) the effect of increased muscle functional length, (2) the effect of surgical detachment, with or without reattachment, and (3) the effect of decreased functional length on the physiological and morphological characteristics of the masseter and temporalis muscles.

Group A-appliance only. Six group A monkeys received the bite-opening appliance and were subjected to no other experimental manipulations except routine radiological examination.

Group $A D$-appliance/detachment. In order to evaluate the effects of muscle detachment on the masseter muscle following stretching, the masseter muscles of eight monkeys were bilaterally surgically detached from their insertions along the ramus of the mandible 1 day after cementation of a bite-opening appliance on the maxillary arch. They were not surgically reattached to the pterygomasseteric sling, but were allowed to form spontaneous reattachments.

Group ADR-appliance/detachment/reat. tachment. An appliance was cemented to the maxillary arch of six animals 1 day prior to surgery and each masseter muscle was detached, then immediately reattached to the pterygomasseteric sling.

Group DR-detached/reattached. The masseter muscles of six animals were bilaterally detached and reattached surgically, but no bite-opening appliance was used. Thus, group DR animals permitted separation of the effects of opened bite from the effects of surgery in the determination of the adaptations in the masseter muscle to altered function.

Group PT--posttreatment. Bite-opening appliances were cemented onto the maxillary arch of five monkeys in group PT, and following a 24-week period of treatment the appliances were removed. This design initiated an experimental period to evaluate the effects of decreased muscle functional length on the morphology and physiology of a muscle previously subjected to chronic stretch.

\section{METHODS}

Surgical procedures

Masseter myotomy. Animals were preanesthetized with an intramuscular injection of phencyclidine hydrochloride $(1.5 \mathrm{mg} / \mathrm{kg})$. 
Pentobarbital sodium $(5 \mathrm{mg} / \mathrm{kg})$ was administered intravenously in the operating room to obtain a surgical level of anesthesia.

An incision approximately $3.5 \mathrm{~cm}$ to $4.0 \mathrm{~cm}$ in length was made through the skin along the inferoposterior border of the mandible. The subcutaneous fat and platysma muscle were sharply dissected to expose the masseter muscle at the gonial angle. Following blunt dissection of the soft tissues surrounding the masseteric fascia, the masseter muscle was incised from the posterior border of the ramus of the mandible and along the inferior mandibular border to the region of the antegonial notch. A periosteal elevator was then used to strip the entire masseter muscle from the inferior border of the ramus proximally to the level of the sigmoid notch. The region was packed with gauze to establish hemostasis and the procedure was repeated on the other side.

In group $\mathrm{AD}$ animals, the masseter muscle was realigned as closely as possible along the ramus, but no attempt was made to reattach it. In group ADR and DR animals, the masseter muscle was reapproximated in its preoperative position and 3-0 chromic suture was used to reattach it. The platysma layer was then closed using 3-0 chromic suture and the skin was closed using 5-0 silk suture.

There were no significant or unusual sequelae in any of the monkeys who underwent surgical intervention. Each resumed her normal dietary behavior within 7 to 10 days following surgery.

Biopsy. Animals were sacrificed at selected times after operative procedures. No animal was sacrificed at less than 4 weeks postoperatively. Regression analysis revealed no significant alterations in measured parameters following 4 weeks; thus adaptations were complete within 4 weeks. At the time of sacrifice, monkeys were preanesthetized with ketamine $(10 \mathrm{mg} / \mathrm{kg} \mathrm{IM})$ followed by an intravenous injection of pentobarbital sodium to induce surgical anesthesia. The right superficial masseter and temporalis muscles were exposed and samples for histochemical and biochemical analyses were excised from standardized sampling sites (Maxwell et al., 1979, 1980a,b) near the anterior and posterior border of the superficial lamina of these muscles. Samples were also obtained from the midline portion of the anterior belly of the digastric muscle. Histochemical samples (approximately $4 \times 4 \times 4 \mathrm{~mm}$ ) were quick frozen in isopentane cooled with dry ice. Muscle samples weighing approxi- mately 300 to $400 \mathrm{mg}$ were prepared for biochemical analysis of oxidative capacity.

Histochemistry. Cross sections $14 \mu \mathrm{m}$ thick were cut from a frozen block in the cryostat at $-20^{\circ} \mathrm{C}$. Sections were incubated for myofibrillar ATPase (Chayen et al., 1973) with or without preincubation for 15 minutes at $\mathrm{pH}$ $10.2-10.4$, succinic acid dehydrogenase (SDH) (Nachlas et al., 1957), and for capillary membrane phosphatase (Maxwell et al., 1977) activities (Figs. $1-3$ ).

Myofibrillar ATPase. Without alkaline preincubation, muscle fibers of the masseter muscle could be differentiated on the basis of the activity of myofibrillar ATPase, but low-activity fibers were nonuniform in reaction intensity (Fig. 2) and some fibers had only slightly less intense ATPase than high-activity fibers. This is in contrast to the excellent differentiation observed in limb muscles and in the temporalis muscle (Maxwell et al., 1980b). Alkaline preincubation inhibited the low-activity fibers such that they became more uniform in reaction intensity and were more easily distinguished from the high-activity fibers. The myofibrillar ATPase activity demonstrated by each type of muscle fiber could be completely inhibited by fixation of sections in calcium formol or by inclusion of parahydroxy-mercuribenzoate (PHMB) in the incubation medium.

A section of each muscle sample incubated for myofibrillar ATPase was projected at $x$ 1,000 and the outlines of individual fibers in each of three or four $25 \mathrm{~cm} \times 40 \mathrm{~cm}$ sample areas were traced. At least 100 fibers were traced from each muscle, and the crosssectional area of each determined by planimetry. Fibers completely within the sample area and fibers which crossed the top and right boundaries and top corners of the sample area were included in the sample. Fibers crossing the other boundaries and corners of the sample area were excluded.

In cat gastrocnemius muscle, muscle fibers with low myofibrillar ATPase contract slowly whereas those with high myofibrillar ATPase contract quickly (Burke et al., 1971). This correlation of physiological and histochemical characteristics has not yet been performed for fibers of the masticatory muscles of rhesus monkeys. Furthermore, histochemical reactions for myofibrillar ATPase, particularly following acid preincubation, differ for masticatory compared to limb muscles of monkeys (Maxwell et al., 1980b). Due to these limita- 

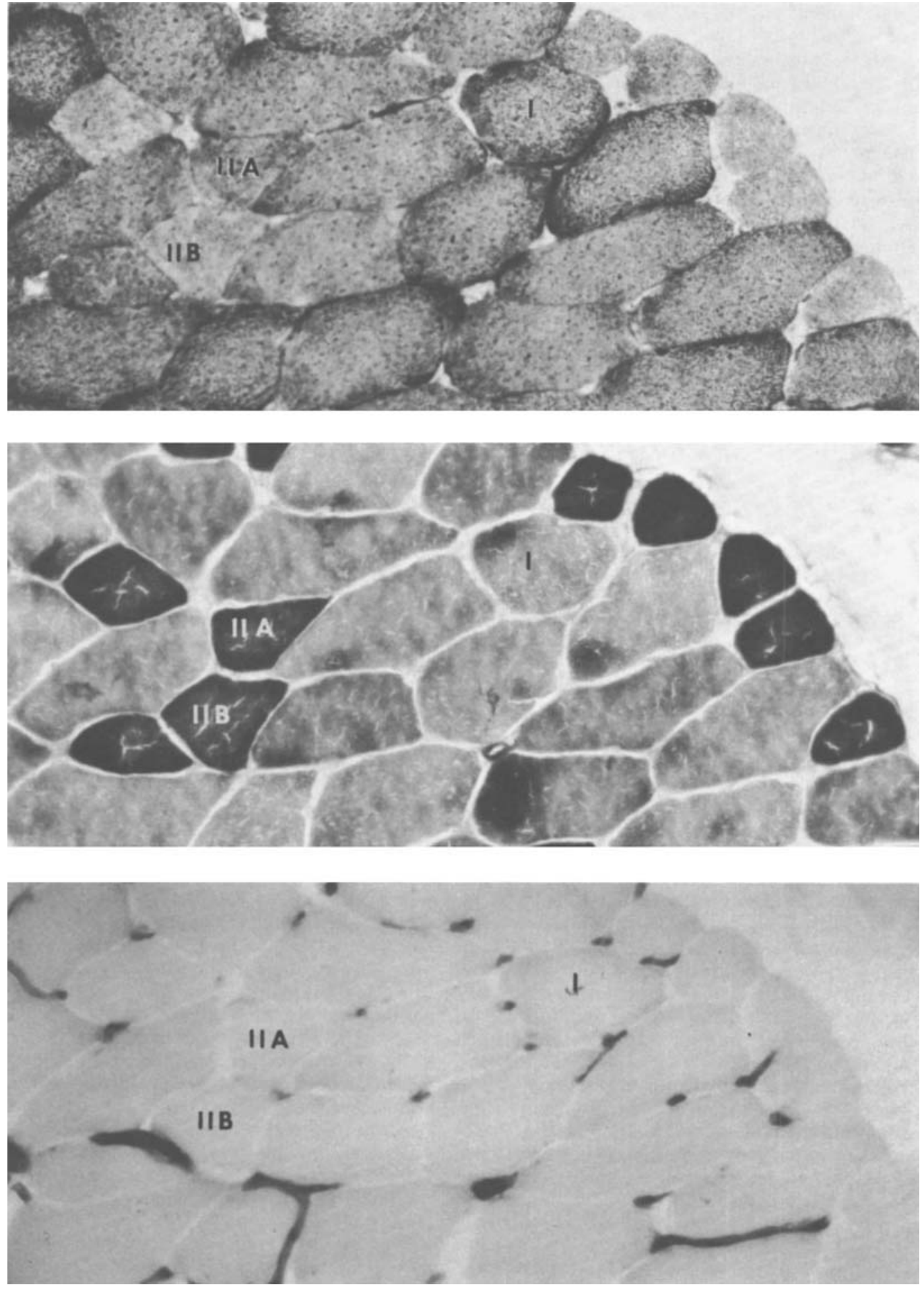
tions, we classified masticatory muscle fibers only as Type I or Type II (Fig. 2) based on myofibrillar ATPase activity (Brooke and Kaiser, 1970), rather than as slow or fast in accordance with Burke et al. (1971), as we had done previously.

$S D H$. A serial section incubated for $\mathrm{SDH}$ activity was then projected, the same sample fibers were identified, and Type II fibers were further classified as Type IIa or Type IIb. Fibers classified IIa had distinct SDH activity and subsarcolemmal aggregates of diformazan, especially near capillaries. Fibers classified IIb had very little SDH activity in subsarcolemmal regions of the fibers. Both localization and intensity of activity were used in determining fiber classification. Type I, Type IIa, and Type IIb fibers were identified (Fig. 1).

Capillarity. A third serial section incubated for capillary membrane phosphatase activity (Fig. 3) was projected and the location of capillaries relative to the same sample fibers were drawn on tracings. The number of capillaries within the sample area, as well as the number of capillaries adjacent to each fiber in the sample, were counted.

Fiber area. Fiber area was determined by planimetry of fiber outlines drawn from projection of sections incubated for myofibrillar ATPase. Mean fiber area, the percentage of each type of fiber in the sample (percent composition), fibers $/ \mathrm{mm}^{2}$, capillaries $/ \mathrm{mm}^{2}$, and capillary to fiber ratio (capillaries $/ \mathrm{mm}^{2} \mathrm{di}$ vided by fibers $/ \mathrm{mm}^{2}$ ) were calculated.

Oxidative capacity. Muscle samples for biochemical procedures were blotted to remove excess moisture, weighed, and homogenized in 19 volumes of ice cold $0.01 \mathrm{M}$ phosphate buffer (pH 7.4) using a Polytron tissue homogenizer. Succinate oxidase activity for duplicate aliquots of homogenate was determined by dif- ferential respirometry, essentially as described by Potter (1964). Protein content of the homogenate was determined by the method of Lowry et al. (1951) and succinate oxidase activity was expressed as $\mathrm{nl}$ of oxygen consumed - mg homogenate protein ${ }^{-1} \cdot$ minute $^{-1}$.

Fiber length. At sacrifice, after removal of histochemical and biochemical samples, the animals were perfused with a fixative solution of neutral-buffered formalin. The heads were removed and placed in $10 \%$ formalin for storage. The masseter and temporalis muscles were then removed intact from the skull. The deep portion of the masseter muscle was separated from the superficial masseter and the muscles were stored in $10 \%$ formalin for at least 1 week. Fixed muscles were then immersed in 30\% nitric acid to digest connective tissue and release muscle fiber bundles (Williams and Goldspink, 1971; Maxwell et al. 1974). The acid was then replaced with $50 \%$ glycerol and small bundles of muscle fibers were carefully teased free. The length of 20 such bundles was determined by measurement under a dissecting microscope fitted with an ocular micrometer. Fibers from these bundles were then mounted in 50\% glycerol and examined at $\times 1,000$ under oil immersion. The length of 10 sarcomere units was determined for at least 20 different fibers. Dividing mean fiber length by mean sarcomere length yields an estimate of the numbers of sarcomeres comprising individual muscle fibers.

Statistical evaluation. Animals were sacrificed at selected intervals greater than 4 weeks after initiation of treatment. When analyzed by linear regression, no significant relationship with the duration of treatment was observed. Therefore, data from all muscles within an experimental group were pooled and statistical measures were calculated. Statistical analyses included analysis of variance to detect significant differences within the

Fig. 1. A photomicrograph of a cross section of an anterior masseter muscle incubated for succinate dehydrogenase activity shows variability in reaction amongst fibers. Selected examples representing three types of muscle fiber are identified for comparison with Figures 2 and 3. Magnification is $\times 200$.

Fig. 2. A photomicrograph of a serial cross section of an anterior masseter muscle incubated for myofibrillar ATPase activity without prior alkaline incubation illustrates Type I (low-activity) and Type II (high-activity) fibers. No fibers with "intermediate" ATPase activity are present in this sample. The same representative fibers are identified as in Figure 1, and illustrate the histochemical profiles of three fiber types. Magnification is $\times 200$.

Fig. 3. Capillarity of a cross section of a serial cross section of an anterior messeter muscle is demonstrated by capillary membrane phosphatase activity. Magnification is $\times 200$. 
grouped data for each variable, and the Newman-Keuls multiple comparison test to identify differences between specific groups. In a few cases it was desirable to pool data into two major groups to test specific hypotheses. In these cases, differences were tested by t-test. Significance was accepted at the $P<0.05$ level.

\section{RESULTS}

\section{Fiber type}

A variable proportion of muscle fibers in the anterior regions of some masseter and temporalis muscles exhibited myofibrillar ATPase activity greater than expected for Type I fibers, but less than the activity of Type II fibers. When fibers with this "intermediate" level of myofibrillar ATPase activity were classified, no consistent pattern for the frequency of occurrence was observed, and many muscles of control and of experimental animals had no fibers with the intermediate level of activity. It was often difficult to distinguish whether a fiber was truly "intermediate" or was a Type I fiber with nonhomogeneous reaction intensity. Therefore, fibers with intermediate myofibrillar ATPase activity were pooled with the Type I fibers.

No difference from control data in the proportion of Type I fibers was observed for muscles in any experimental group. Although Type II fibers in muscles of experimental animals had less intense SDH activity than muscle fibers of control animals, many Type II fibers retained the pattern of localization of SDH activity classifying them as Type IIa. Thus, in masticatory muscles of experimental relative to control animals, there was reduced oxidative capacity but no significant alteration in the proportion of muscle fibers of the three classifications (Table 1).

\section{Oxidative capacity}

Succinate oxidase activity of muscle homogenates (Table 2) supports the observations on the histochemical demonstration of SDH activity. Masseter muscles of groups A, AD, ADR, and DR had significantly less succinate oxidase activity than the muscles of controls or Group PT animals. In anterior portions of temporalis muscles from animals in which an appliance was present at sacrifice (group A, $A D, A D R$ ) succinate oxidase activity was less than for the muscles of animals which did not (control and groups DR, PT). No differences from control values were observed for the succinate oxidase activity of the posterior tem- poralis or diagastric muscles in any experimental group.

\section{Fiber area}

The mean cross-sectional areas of Type I fibers in group ADR animals was significantly reduced relative to controls in the anterior masseter muscle (Table 1). This effect did not occur in posterior masseter, anterior or posterior temporalis, or digastric muscles of these animals.

\section{Capillarity}

Determination of the number of adjacent capillaries for individual muscle fibers revealed no significant alteration from control value for the muscle of the experimental groups (Table 3). Capillary density values (Table 3 ) were occasionally greater than the control value, reflecting changes in mean fiber area of the muscle samples. Capillarity seemed to be only minimally affected by the experimental procedures.

\section{Fiber length}

The number of sarcomeres comprising muscle fibers of deep and superficial masseter muscles was not significantly altered from control values by experimental procedures, whether the functional length of the masseter was increased by the appliance, the masseter was detached, or detached and reattached (Table 4). Due to trends in opposing directions, fibers of masseter muscles were longer in groups PT than ADR animals. Significantly more sarcomeres per temporalis muscle fiber were observed in those animals which had surgical manipulation of masseter muscle in addition to a bite-opening appliance (groups AD, ADR) than in those animals which did not (control, A, DR, PT). Neither surgical manipulation of the masseter alone (group DR) nor the appliance alone (group A) were sufficient to induce significant adaptation from control, but a combination of the two stimuli was required for the effect.

\section{DISCUSSION}

Histochemical demonstration of myofibrillar ATPase activity of masticatory muscle fibers does not exactly fit the pattern normally seen for three types of muscle fibers in limb muscles (Maxwell et al., 1980a). Specifically, when incubated without prior treatment, some of the fibers in masticatory muscles demonstrate an activity relatively greater than usually seen for Type I fibers, but less than for 
TABLE 1. Percentage Composition and Mean Fiber Area For Masseter, Temporalis, and digastric muscles (data are mean \pm $S E M)$

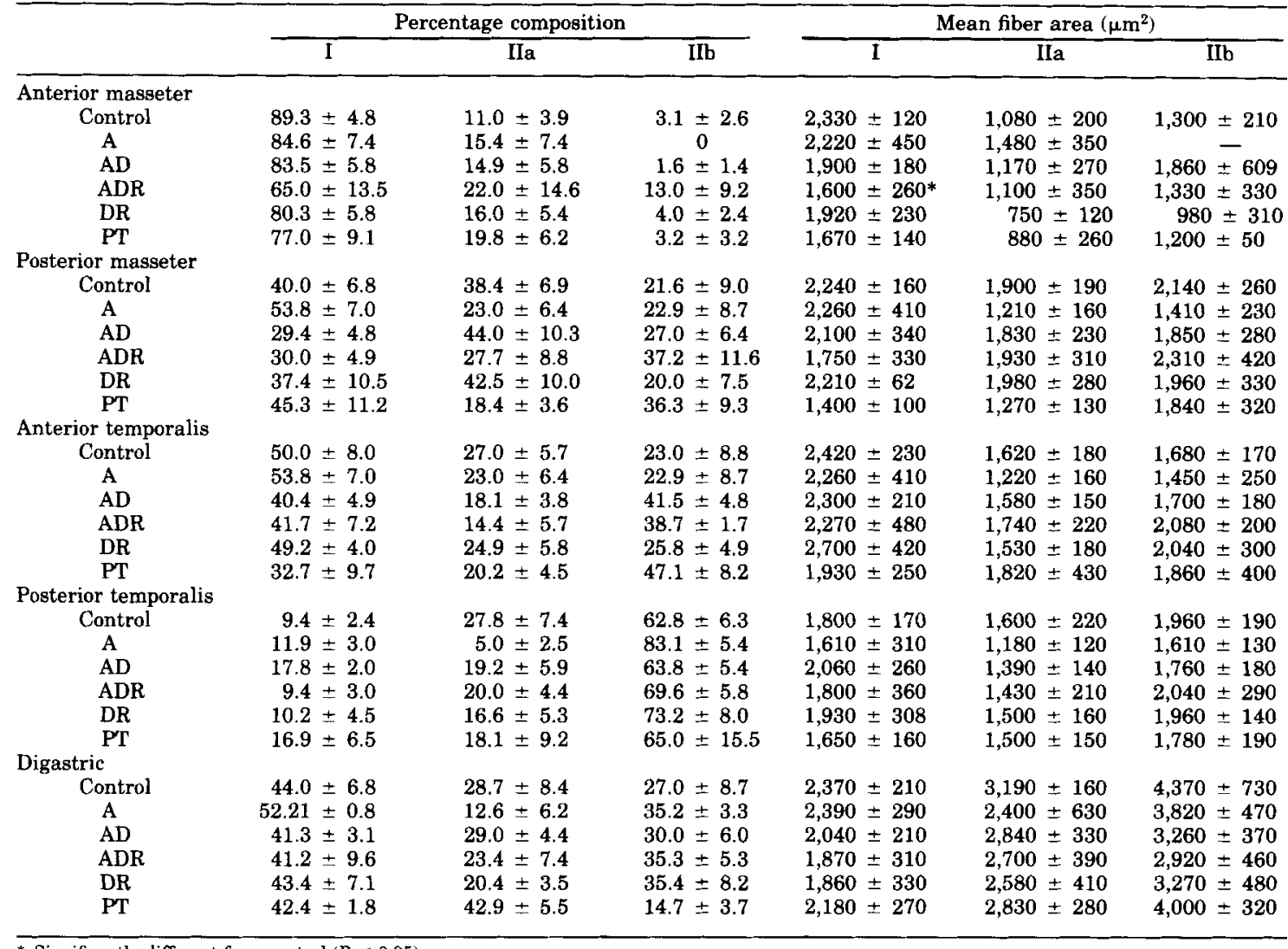

* Significantly different from control $(P<0.05)$.

TABLE 2. Succinate oxidase activity of muscle homogenates $\left(\mathrm{nl} \mathrm{O}_{2} / \mathrm{mg}\right.$ homogenate protein $-1 /$ min $^{-1}$ ) (data are mean \pm SEM)

\begin{tabular}{llclrrr}
\hline & \multicolumn{2}{c}{ Masseter } & & \multicolumn{2}{c}{ Temporalis } & \\
\cline { 2 - 3 } & Anterior & Posterior & & Anterior & Posterior & Digastric \\
\hline Control & $441 \pm 24$ & $416 \pm 33$ & & $330 \pm 24$ & $175 \pm 26$ & $218 \pm 126$ \\
A & $170 \pm 28^{*}$ & - & $144 \pm 31^{*}$ & $76 \pm 12$ & $169 \pm 32$ \\
AD & $205 \pm 28^{*}$ & - & & $180 \pm 28^{*}$ & $106 \pm 21$ & $234 \pm 38$ \\
ADR & $158 \pm 39^{*}$ & - & & $177 \pm 42^{*}$ & $134 \pm 38$ & $227 \pm 77$ \\
DR & $225 \pm 51^{*}$ & - & & $212 \pm 36$ & $85 \pm 13$ & $136 \pm 45$ \\
PT & $387 \pm 37$ & - & $296 \pm 82$ & $171 \pm 44$ & $344 \pm 27$ \\
\hline
\end{tabular}

* Significantly different from control or PT value $(P<0.05)$,

Type II fibers. It has been suggested that fibers which exhibit an intermediate intensity of myofibrillar ATPase activity may be transitional between Type I and Type II fiber types which are responding to alterations in functional demand (Ringqvist, 1973, 1974, 1977;
Kugelberg, 1976; Maxwell et al., 1980b). According to this hypothesis greater proportions of "intermediate" fibers might be expected following alterations of muscle function which result in significant differences in the proportion of Type I and Type II fibers. However, in 
$T A B L E$ 3. Capillary of masseter, temporalis, and digastric muscles (data are mean $\pm S E M$ )

\begin{tabular}{|c|c|c|c|c|c|}
\hline & \multicolumn{3}{|c|}{$\begin{array}{l}\text { Number of adjacent } \\
\text { capillaries }\end{array}$} & \multirow{2}{*}{$\begin{array}{c}\text { Capillaries } \\
\text { per } \mathrm{mm}^{2}\end{array}$} & \multirow{2}{*}{$\begin{array}{l}\text { Capillary to muscle } \\
\text { fiber ratio }\end{array}$} \\
\hline & 1 & IIa & IIb & & \\
\hline \multicolumn{6}{|c|}{ Anterior masseter } \\
\hline Control & $5.29 \pm 0.43$ & $3.49 \pm 0.70$ & $3.09 \pm 0.91$ & $863 \pm 68$ & $2.30 \pm 0.21$ \\
\hline A & $5.16 \pm 0.46$ & $3.63 \pm 0.69$ & - & $793 \pm 94$ & $1.79 \pm 0.610$ \\
\hline $\mathrm{AD}$ & $5.79 \pm 0.74$ & $3.71 \pm 0.97$ & - & $972 \pm 97$ & $2.06 \pm 0.25$ \\
\hline ADR & $3.90 \pm 0.50$ & $2.99 \pm 0.43$ & $3.34 \pm 0.48$ & $825 \pm 73$ & $1.38 \pm 0.22$ \\
\hline DR & $6.01 \pm 0.76$ & $3.57 \pm 0.37$ & $3.02 \pm 0.50$ & $931 \pm 135$ & $2.10 \pm 0.33$ \\
\hline PT & $4.03 \pm 0.46$ & $2.37 \pm 0.24$ & - & $778 \pm 153$ & $1.37 \pm 0.17$ \\
\hline \multicolumn{6}{|c|}{ Posterior masseter } \\
\hline Control & $4.59 \pm 0.47$ & $3.60 \pm 0.49$ & $3.73 \pm 0.72$ & $566 \pm 82$ & $1.45 \pm 0.17$ \\
\hline A & $4.35 \pm 0.38$ & $3.48 \pm 0.26$ & $3.40 \pm 0.22$ & $753 \pm 89$ & $1.49 \pm 0.08$ \\
\hline $\mathrm{AD}$ & $4.76 \pm 0.56$ & $4.14 \pm 0.82$ & $4.13 \pm 0.87$ & $778 \pm 38$ & $1.75 \pm 0.28$ \\
\hline ADR & $4.02 \pm 1.22$ & $3.99 \pm 1.23$ & $3.55 \pm 0.72$ & $932 \pm 99^{*}$ & $2.15 \pm 0.39$ \\
\hline DR & $5.45 \pm 0.77$ & $4.94 \pm 0.43$ & $4.07 \pm 0.81$ & $991 \pm 91^{*}$ & $2.36 \pm 0.21$ \\
\hline $\mathrm{PT}$ & $4.20 \pm 0.50$ & $3.24 \pm 0.19$ & $3.06 \pm 0.29$ & $720 \pm 137$ & $1.26 \pm 0.22$ \\
\hline \multicolumn{6}{|c|}{ Anterior temporalis } \\
\hline Control & $5.38 \pm 0.48$ & $3.35 \pm 0.49$ & - & $645 \pm 90$ & $1.86 \pm 0.27$ \\
\hline A & $4.52 \pm 0.44$ & $3.15 \pm 0.47$ & $3.30 \pm 0.40$ & $746 \pm 96$ & $1.60 \pm 0.17$ \\
\hline $\mathrm{AD}$ & $5.44 \pm 0.56$ & $3.23 \pm 0.34$ & $3.52 \pm 0.25$ & $823 \pm 68$ & $1.71 \pm 0.12$ \\
\hline ADR & $4.49 \pm 0.63$ & $3.66 \pm 0.65$ & $3.59 \pm 0.10$ & $608 \pm 126$ & $1.34 \pm 0.14$ \\
\hline $\mathrm{DR}$ & $5.93 \pm 1.07$ & $4.03 \pm 0.33$ & $4.12 \pm 0.39$ & $677 \pm 93$ & $1.64 \pm 0.21$ \\
\hline$P T$ & - & - & - & - & - \\
\hline \multicolumn{6}{|c|}{ Posterior temporalis } \\
\hline $\begin{array}{c}\text { Control } \\
\text { A }\end{array}$ & $\begin{array}{c}3.97 \pm 0.36 \\
-\end{array}$ & $\begin{array}{c}3.31 \pm 0.27 \\
-\end{array}$ & $\begin{array}{c}2.84 \pm 0.26 \\
-\end{array}$ & $\begin{array}{c}567 \pm 37 \\
-\end{array}$ & $\begin{array}{c}1.35 \pm 0.14 \\
-\end{array}$ \\
\hline AD & $5.12 \pm 0.75$ & $3.70 \pm 0.26$ & $3.42 \pm 0.34$ & $962 \pm 20$ & $1.67 \pm 0.16$ \\
\hline $\mathrm{ADR}$ & - & - & - & - & - \\
\hline DR & $4.00 \pm 0.32$ & $4.00 \pm 0.33$ & $3.51 \pm 0.25$ & - & - \\
\hline PT & - & - & - & - & - \\
\hline \multicolumn{6}{|l|}{ Digastric } \\
\hline Control & $\begin{array}{c}4.88 \pm 0.65 \\
-\end{array}$ & $\begin{aligned} 6.04 & \pm 0.08 \\
& -\end{aligned}$ & - & $\begin{array}{c}646 \pm 103 \\
-\end{array}$ & $\begin{array}{c}2.11 \pm 0.10 \\
\leq\end{array}$ \\
\hline $\begin{array}{l}\mathrm{AD} \\
\mathrm{ADR}\end{array}$ & $\begin{array}{c}4.83 \pm 0.92 \\
-\end{array}$ & $\begin{array}{c}5.45 \pm 1.30 \\
-\end{array}$ & $\begin{array}{c}4.60 \pm 0.68 \\
\simeq\end{array}$ & $\begin{array}{c}1017 \pm 109 \\
-\end{array}$ & 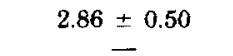 \\
\hline $\begin{array}{l}\text { DR } \\
\text { PT }\end{array}$ & $\begin{array}{c}4.37 \pm 0.86 \\
-\end{array}$ & $4.75 \pm 1.29$ & $4.51 \pm 0.61$ & $873 \pm 18$ & $2.30 \pm 0.49$ \\
\hline
\end{tabular}

* Significantly different from control $(P<0.05)$.

the current research no differences in the proportion of Type I and Type II fibers were found between any of the experimental groups and controls. Thus, if fibers with "intermediate" myofibrillar ATPase activity are transitional fibers in which the type of myosin is altering, it could be that at any point in time a small but equal number of fibers are converting from Type I to Type II as from Type II to Type I. Alternatively, it is possible that the muscles of mastication contain a different fiber type which is actually intermediate in myofibrillar ATPase activity, or which requires different conditions for optimal expression of myofibrillar ATPase. This possibility receives added impetus from a recent analysis demonstrating significant differences in $\mathrm{pH}$ lability of myofibrillar ATPase between fibers of masticatory and limb muscles from rhesus monkeys (Maxwell et al., 1980b).

Since fibers with "intermediate" myofibrillar ATPase activity were not observed in every muscle, pooling of fibers which have lower myofibrillar ATPase activity than Type II fibers into one group as Type I fibers simplifies results. This approach is justified because (1) the intermediate level of activity more closely resembles the activity of Type I than Type II fibers; (2) preincubation at $\mathrm{pH}$ 10.2-10.4 partially inhibits the activity of these fibers, thus displaying alkaline lability characteristic of Type I fibers (Brooke and Kaiser, 1970); (3) there was no difference in capillarity, oxidative capacity, or fiber area between the fibers with intermediate ATPase activity and Type I fibers; and (4) the sum of the proportions of 
TABLE 4. Number of sarcomeres per muscle fiber (data are mean \pm $S E M)$

\begin{tabular}{llll}
\hline & $\begin{array}{c}\text { Superficial } \\
\text { masseter }\end{array}$ & $\begin{array}{c}\text { Deep } \\
\text { masseter }\end{array}$ & Temporalis \\
\hline Control & $5,908 \pm 327$ & $4,241 \pm 646$ & $7,149 \pm 471$ \\
A & $5,758 \pm 434$ & $4,410 \pm 427$ & $7,224 \pm 472$ \\
AD & $6,440 \pm 333$ & $4,433 \pm 451$ & $7,943 \pm 396$ \\
ADR & $5,226 \pm 506^{*}$ & $4,388 \pm 451$ & $8,468 \pm 421$ \\
DR & $5,875 \pm 372$ & $4,972 \pm 519$ & $6,700 \pm 510$ \\
PT & $\mathbf{8 , 2 0 0 \pm 1 9 0 6}$ & $\mathbf{4 , 3 8 0 \pm 9 4 1}$ & $7,287 \pm 656$ \\
C, DR, PT & & & $7,038 \pm 310$ \\
AD, ADR & & & $8,162 \pm 288^{* *}$ \\
\hline
\end{tabular}

* Group ADR significantly different from group PT.

** Pooled data for groups AD, ADR significantly different from pooled data for groups C, DR, PT $(P<0.05)$.

fibers with intermediate activity and Type I fibers was consistent both within and between groups.

Several recent histochemical studies of the muscles of mastication demonstrate changes in fiber cross-sectional area and in percentage composition of fiber types in response to alterations in functional demand. An analysis of the histochemical characteristics of the masseter and temporalis muscles in normal juvenile and adult rhesus monkeys, for example, found significant differences in percentage composition and cross-sectional area of specific fiber types between juveniles and adults, and between adult males and females (Maxwell et al., 1979). It was suggested that these variations in histochemical properties of the muscles of mastication actually derive from alteration in jaw function associated with the developing occlusion and secondary sex characteristics of the adult dentofacial complex. In a study of denture wearers, Ringqvist (1974) noted a reduced percentage and reduced crosssectional area of Type II fibers in the temporalis muscle of individuals who described their dentures as deficient, relative both to individuals with a natural dentition and to individuals with well-fitted dentures. An experimental study of the masseter and temporalis muscles of female monkeys who were edentulous for a period of 4.5 years (Maxwell et al., 1980a) demonstrated a pronounced change in histochemical composition and fiber area in the edentulous monkeys relative to controls. The edentulous animals had significantly fewer Type I fibers and more Type IIb fibers in the posterior masseter and anterior and posterior temporalis. Moreover, the Type I fibers were smaller in mean cross-sectional area than Type IIb fibers at all sample sites-ex- actly the opposite of the situation in the control female animals.

Nevertheless, failure to observe an alteration in the proportion of the different types of muscle fibers with the experimental techniques in this report is not totally unexpected. Limb muscles respond to increased functional length due to growth without alteration of myofibrillar ATPase (Maxwell et al,, 1973). Furthermore, no alteration of myofibrillar A'TPase was observed following an endurance training program (Maxwell et al., 1973). Thus, there are examples in the literature of altered functional length or altered activity level which do not cause changes in myofibrillar ATPase. Chronic electrical stimulation experiments suggest that activity induced by bursts of impulses at a frequency of 10/sec once each second will induce the transformation of myosin from Type II to Type I (Salmons and Sreter, 1976). Thus, the experimental procedures in the current study may not have significantly altered the pattern of impulses to the muscle from motor neurons. It is perhaps the frequency of impulses within a burst of activity, rather than the frequency of bursts of activity, which is important in the regulation of the type of myosin in muscle fibers. If so, changes in overall activity level without change in the frequency of impulses within bursts would result in no change in myofibrillar ATPase.

Oxidative capacity of muscle fibers (Maxwell et al., 1973) does respond to changes in the overall level of activity. Reduced intensity of SDH activity in muscle fibers and the reduced succinate oxidase activity in muscle homogenates in groups $\mathrm{A}, \mathrm{AD}, \mathrm{ADR}$, and $\mathrm{DR}$ all suggest a reduction relative to control in the overall activity of these muscles following 
placement of an intraoral appliance or masticatory muscle surgery. As demonstrated by the data from group P'T, the effect due to the appliance is reversible, and return toward control values occurs following removal of the appliance. Lack of significant change in the oxidative capacity of digastric muscle demonstrates that the effect of the experimental procedures is selective, occurring in specific muscles, and that demonstrated differences from control represent effects of experimental procedures rather than general effects on muscle resulting from age, time in captivity, or handling of animals.

The placement of a bite-opening appliance in the mouth causes masticatory muscle fibers to function at greater than optimal length and at decreased mechanical advantage due to the altered position of the mandible (Carlson, 1977). However, no change in mean fiber area was observed in response to the stretch induced by the appliance alone (group A). Surgical detachment of the insertion of masseter muscle relieves the stretch of the muscle. A decrease relative to control of Type I fiber cross-sectional area was observed in those animals in which the masseter was surgically detached and reattached (group ADR).

Muscle fibers could adapt to alterations in functional length by addition or deletion of sarcomeres to muscle fibers, by rearrangement of muscle fiber orientation, or both. In limb muscles immobilized by plaster casts, Tabary et al. (1972) and Goldspink et al. (1974) have demonstrated addition of sarcomeres to muscle fibers when immobilization length exceeded the normal rest length and loss of sarcomeres when muscles were immobilized at less than rest length. These changes in sarcomere number in response to functional length changes occur within 4 weeks even in denervated muscles (Goldspink et al., 1974). Inclusion of the appliance in the mouths of group A animals produced approximately a 15\% increase in functional length of masseter (Carlson, 1977) and temporalis muscles. However, no alteration in the number of sarcomeres per muscle fiber were observed in group A animals. Animals in group A were smaller $(4.82 \pm 0.32 \mathrm{~kg})$ than control animals $(6.13 \pm 0.42 \mathrm{~kg})$. Perhaps this masked an alteration of fiber length in this group. This suggestion receives support from the observation of greater than control length of masseter muscles in group PT, which had also been subjected to stretch by the appliance. No alteration of the number or sarcomeres per muscle fiber was observed in temporalis muscles of control or group A, DR, PT animals. Thus, it appears that when both masseter and temporalis muscles can contribute to the support and function of the mandible, any adaptation to the increased functional length must have occurred through geometrical rearrangement of temporalis muscle fibers. However, when the masseter muscle insertion was surgically detached and the masseter muscle was less able to contribute to mandibular movement and support, sarcomeres were added to the fibers of temporalis muscle. This effect occurred only in response to the combined stimulus of the appliance and detachment of the masseter, whether or not masseter was surgically reattached (groups AD, ADR). Neither the appliance alone (group A), nor surgical detachment and reattachment of masseter muscle alone (group DR), were effective. The average 15\% increase in the number of sarcomeres of temporalis muscle fibers in groups $A D$ and ADR corresponds well to the increase in functional length of the temporalis muscle.

\section{CONCLUSIONS}

Alterations in the functional length of the masseter and temporalis muscles, with and without masseter myotomy, resulted in only subtle morphological and physiological adaptations. Nevertheless, certain general conclusions can be drawn from the data which contribute to our understanding of muscle adaptation following surgical interventions or alterations in length.

1. Stretching the masseter and temporalis muscles within physiological limits did not significantly alter the proportion of fiber types; however, it did appear to cause a reduction in the oxidative capacity of Type I and Type IIa fibers. Removal of the bite-opening appliance, thus alleviating the stretch of the masseter and temporalis muscles, resulted in a "recovery" of the potential for oxidative phosphorylation to within normal limits.

2. Fibers with intermediate intensity of myofibrillar ATPase activity were no more prevalent in muscles of experimental animals than in controls.

3 . Surgical detachment of the masseter muscle with surgical reattachment resulted in a significant decrease in the mean cross-sectional area of Type I fibers in the anterior masseter muscle. These data indicate that the cross-sectional area of Type I fibers, which are probably most active during postural activity and sustained mastication, is most sensitive to alterations in jaw function. 
4. There was minimal alteration of muscle capillarity, regardless of the experimental protocol followed. Thus, it appears that neither alteration in functional length nor surgical intervention of the sort described in this analysis have any significant direct effect on the capillarity of masseter and temporalis muscles.

5 . The length of individual fibers within the temporalis muscle increased following muscle lengthening in conjunction with masseter myotomy (i.e., groups AD and ADR), probably due to an addition of sarcomeres at the terminal ends of the fibers. There was no increase in fiber length within either the masseter muscle or the temporalis muscle following muscle stretching alone (group A); nor was there any increase in fiber length in either muscle associated with masseter myotomy alone (group DR). These data suggest that compensatory activity of the temporalis muscle (1) following masseter myotomy and (2) from a length which is greater than its normal resting length, lead to longitudinal growth of the temporalis muscle fibers. Increase in the length of the temporalis fibers through addition of sarcomeres reestablishes the optimal overlap between the actin and myosin filaments within each sarcomere, increasing the tension developed by temporalis muscle fibers at the longer length and in the absence, however transient, of masseter function.

\section{ACKNOWLEDGMENTS}

This work was supported by United States Public Health Service grants DE 04227 and by contract DE 52748 from the National Institute of Dental Research, National Institutes of Health.

\section{LITERATURE CITED}

Brooke, M.H., and K.K. Kaiser (1970) Muscle fiber types: How many and what kind? Arch. Neurol., 23: 369-379.

Burke, R.E., P.N. Levine, F.E. Zajac, III, P. Tsairis, and W.K. Engel (1971) Mammalian motor units: Physiological correlates of three types in cat gastrocnemius. Science, 174: 709-712.

Carlson, D.S. (1977) Condylar translation and the function of the superficial masseter muscle in the rhesus monkey (M. Mulatta). Am. J. Phys. Anthropol., 47: 53-63.

Chayen, J., L. Bitensky, and R.G. Butcher (1973) Practical Histochemistry. John Wiley and Sons, New York, pp. $122-124$.

Christiansen, R.L. (1975) Research in orthognathic surgery-an NIDR state-of-the-art workshop. J. Oral Surg., 33: $907-920$.

Goldspink, G., C. Tabary, J.C. Tabary, C. Tardieu, and G. Tardieu (1974) Effect of denervation on the adaptation of sarcomere number and muscle extensibility to the functional length of the muscle. J. Physiol. (Lond.), 236: $733-742$
Hurme, V.O., and E.G. Van Wagenen (1961) Basic data on the permanent teeth in the rhesus monkey. Proc. Am. Philos. Soc., 105: 105-140.

Kugelberg, E. (1976) Adaptive transformation of rat soleus motor units during growth. J. Neurol. Sci., 27: 269-289.

Lowry, O.H., N.J. Rosebrough, A.L. Faro, and R.J. Crandall (1951) Protein measurement with the Folin phenol reagent. J. Biol. Chem., 193: 265-275. Arch. Neurol., 23: 369-379.

Maxwell, L.C., J.A. Faulkner, and D.A. Lieberman (1973) Histochemical manifestations of age and endurance training in skeletal muscle fibers. Am. J. Physiol., 344: 356-361.

Maxwell, L.C., J.A. Faulkner, and G.J. Hyatt (1974) Estimation of the number of fibers in guinea pig skeletal muscles. J. Appl. Physiol., 37: 259-264.

Maxwell, L.C., J.K. Barclay, D.E. Mohrman, and J.A. Faulkner (1977) Physiological characteristics of skeletal muscles of dogs and cats. Am. J. Physiol., 233: C14-C18.

Maxwell, L.C., D.S. Carlson, J.A. MeNamara, Jr, and J.A. Faulkner (1979) Histochemical characteristics of the masseter and temporalis muscles of the rhesus monkey (Macaca Mulatta). Anat. Rec., 193: 389-401.

Maxwell, L.C., J.A. McNamara, Jr., P.S. Carlson, and J.A. Faulkner (1980a) Histochemical characteristics of fibers of masseter and temporalis muscles of edentulous rhesus monkeys (Macaca Mulatta) Arch. Oral Biol., Arch Oral Biol 25: 87-93.

Maxwell, L.C., D.S. Carlson, and C.E. Brangwyn (1980b) Lack of acid reversal of myofibrillar ATPase in masticatory muscles fibers of rhesus monkeys. Histochem J. 12: 209-219.

McNamara, J.A., Jr. (1975) An experimental study of increased vertical dimension in the growing face. Am. J. Orthod., 71: 382-395.

McNamara, J.A., Jr., D.S. Carlson, G.M. Yellich, and R.P. Hendricksen (1978) Musculoskeletal adaptation following orthognathic surgery. In: Muscle Adaptation in the Craniofacial Region. D.S. Carlson and J.A. McNamara, Jr., eds. Center for Human Growth and Development, The University of Michigan, Ann Arbor, Michigan. pp. 91-132.

Nachlas, M., M.K. Tsou, E. deSouza, C. Cheng, and A.M. Seligman (1957) Cytochemical demonstration of succinic dehydrogenase by the use of a new p-nitrophenyl substituted ditetrazole. J. Histochem. Cytochem., 5: 420-436.

Potter, V.R. (1964) The homogenate technique. In: Manometric Techniques. W.W. Umbreit, R.H. Burris, and J.F. Stauffer, eds. Burgess Publishing, Minneapolis, pp. 159-176.

Ringqvist, M. (1973) Histochemical enzyme profiles of fibers in human masseter muscles with special regard to fibers with intermediate myofibrillar ATPase reaction. J. Neurol. Sci., 18: 133-141.

Ringquist, M. (1974) Fiber types in human masticatory muscles. Relation to function. Scand. J. Dent. Res., 82: 333-355.

Ringqvist, M., I. Ringqvist, and L.A. Thornell (1977) Differentiation of fibers in human masseter, temporalis and biceps brachii muscles. J. Neurol. Sci., 32: 265-273.

Salmons, S., and F.A. Sreter (1976) Significance of impulse activity in the transformation of skeletal muscle type. Nature, 263: 30-34.

Tabary, J.C., C. Tabary, C. Tardieu, G. Tardieu, and G. Goldspink (1972) Physiological and structural changes in the cat's soleus muscle due to immobilization at different lengths by plaster casts. J. Physiol. (Lond.), 224: 231-244.

Williams, P.E., and G. Goldspink (1971) Longitudinal growth of muscle fibers. J. Cell. Sci., 9: 751-767. 